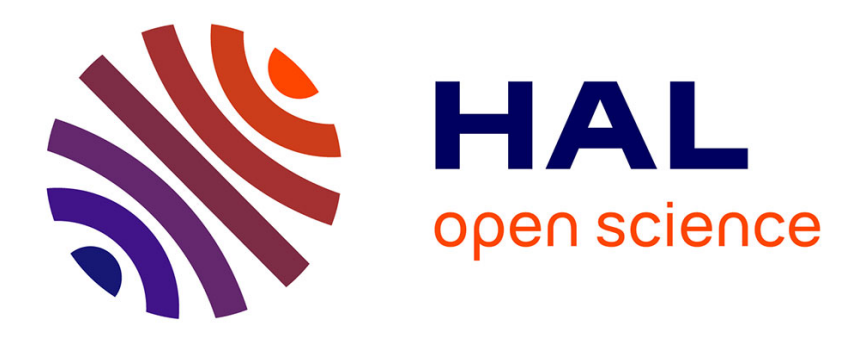

\title{
Drag reduction of a bluff body using adaptative control methods
}

\author{
Jean-François Beaudoin, Olivier Cadot, Jean-Luc Aider, José-Eduardo \\ Wesfreid
}

\section{- To cite this version:}

Jean-François Beaudoin, Olivier Cadot, Jean-Luc Aider, José-Eduardo Wesfreid. Drag reduction of a bluff body using adaptative control methods. Physics of Fluids, 2006, 18, pp.085107. 10.1063/1.2236305 . hal-00173172

\section{HAL Id: hal-00173172 \\ https://hal.science/hal-00173172}

Submitted on 26 Nov 2017

HAL is a multi-disciplinary open access archive for the deposit and dissemination of scientific research documents, whether they are published or not. The documents may come from teaching and research institutions in France or abroad, or from public or private research centers.
L'archive ouverte pluridisciplinaire HAL, est destinée au dépôt et à la diffusion de documents scientifiques de niveau recherche, publiés ou non, émanant des établissements d'enseignement et de recherche français ou étrangers, des laboratoires publics ou privés. 


\title{
Drag reduction of a bluff body using adaptive control methods
}

\author{
Jean-François Beaudoin ${ }^{\text {a) }}$ \\ Department of Research and Innovation, PSA Peugeot-Citroën, 2 route de Gisy, \\ 78943 Vélizy-Villacoublay, France \\ Olivier Cadot \\ Unité de Mécanique, Ecole Nationale Supérieure de Techniques Avancées, Chemin de la Hunière, \\ 91761 Palaiseau Cedex, France \\ Jean-Luc Aider \\ Department of Research and Innovation, PSA Peugeot-Citroën, 2 route de Gisy, \\ 78943 Vélizy-Villacoublay, France \\ José-Eduardo Wesfreid \\ Physique et Mécanique des Milieux Hétérogènes, Ecole Supérieure de Physique et Chimie Industrielles \\ (PMMH UMR 7636-CNRS-ESPCI), 10 rue Vauquelin, 75231 Paris Cedex 5, France
}

A classical actuator is used to control the drag exerted on a bluff body at large Reynolds number $(\operatorname{Re}=20000)$. The geometry is similar to a backward-facing step whose separation point is modified using a rotating cylinder at the edge. The slow fluctuations of the total drag are directly measured by means of strain gauges. As shown by visualizations, the actuator delays the separation point. The size of the low-pressure region behind the body is decreased and the drag reduced. It is found that the faster the rotation of the cylinder, the lower the drag. In a first study, the goal of the control is for the system to reach a drag consign predetermined by the experimentalist. The control loop is closed with a proportional integral correction. This adaptive method is shown to be efficient and robust in spite of the large fluctuations of the drag. In the second method, the system finds itself its optimal set point. It is defined as the lowest cost of global energy consumption of the system (drag reduction versus energy used by the actuator). For this purpose, an extremum seeking control method is applied in order to deal with the large background noise due to turbulence. It consists in a synchronous detection of the response measured in the drag measurements to a modulation of the actuator. The phase shift and amplitude of the modulation estimate the local gradient of the total energy function. With this gradient estimation, the system goes to the minimum of global power consumption by itself. The system is found to be also robust and reacts successfully to changes of the external mean flow. This experiment attests to the real efficiency of local active control in reducing autonomously the global energy consumption of a system under turbulent flow.

\section{INTRODUCTION}

Flow control has become a major subject in both academic and industrial research lying on the intersection of physics, mathematics, and numerical methods. ${ }^{1,2}$ From an academic point of view, it is an exciting theoretical and experimental problem implying a deep understanding of the flow and its dynamics.

A control device is generally designed to operate a system in a desired manner. In the case of flow control, it often consists in modifying the flow conditions by creating perturbations in the boundary conditions in order to improve aerodynamic or hydrodynamic performances. Three control strategies are available at this point.

First, passive control is based on placing motionless disturbances that are known to have a favorable effect on the flow (it is the case of dimples on a golf ball ${ }^{3}$ ).

Next, active control aims at providing an even more fa-

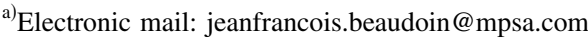

vorable effect, but requires energy input. ${ }^{3-5}$ It often lies on the use of an actuator that creates time-dependent boundary conditions. The latter are defined $a$ priori and without paying regard to external conditions. For instance, Greenblatt and Wygnanski ${ }^{6}$ propose to control separation by periodic excitation using oscillatory blowing and suction or flapping foil.

The third strategy is called feedback control and allows the system to fit to the flow time evolution. ${ }^{7-15}$ The actuator is then connected to one or several sensors so that the activation of the perturbation is a function of some information given by wall, bulk, or global measurements. The elaboration of this function, called the controller design, is a crucial issue for the efficiency of the control loop. Several technical and theoretical problems have to be overcome to achieve an experimental closed-loop flow control. One of them is related to the short time scales characterizing turbulent flows, as detailed in the following.

From a general point of view, closed-loop control is supposed to drive in real time a dynamical system on a predetermined trajectory in the phase space. In many practical 
situations, one can use feedback control to allow a system to keep autonomously its optimal set point. In the case of the control of turbulent flows whose external conditions are stable (constant free-stream velocity, for instance), one often tries to control the dynamics of the coherent structures: vortices distribution in a bluff-body wake, ${ }^{14}$ localization of a separation point, ${ }^{16}$ or longitudinal structures in a turbulent boundary layer. ${ }^{12}$ In these cases, the detection of the structures and the activation of the actuator must be realized as fast as the characteristic times of the structures. It implies a control system including the sensor(s), the actuator(s), and the algorithm efficient enough to deal with high-frequency inputs and outputs. In turbulent flows, which is our case, the characteristic frequencies are large (a few hundred Hertz) and would require very fast actuators such as MEMS (microelectro-mechanical systems ${ }^{17,18}$ ) for experimental control. Nevertheless, there are many realistic and important situations in which external conditions are likely to change, namely the incidence of a wing, modification of the freestream velocity, and the yaw angle of a bluff-body. These phenomena occur on much longer time scales than those of the flow proper fluctuations and have a major influence on the global dynamics of the flow. Considering this point of view, one can see that it becomes realistic to use more "conventional" mechanical actuators for flow control in the case of time-dependent external conditions. We will use this point of view in the following to make the demonstration of the efficiency of feedback control on our system.

Real time is then a first obstacle for experimental feedback turbulent flow control. The second challenge is more theoretical: even if we have an actuator fast enough, we have to find the right algorithm to close the loop (controller design). The search for the right algorithm is different whether we know the governing equations (or reduced-order model) of the dynamical system or only a few of its properties. In the first case, it is possible to anticipate the effect of the control on the dynamics of the system and then to foresee its "longer time" evolution: we then speak about predictive control. $^{1,7,14}$ This method is the most efficient since it may provide an optimal feedback law. Yet it requires knowledge of the future of the system, which seems to be unrealistic for turbulent flows (especially for experiments). We then choose to turn to another approach, called adaptive control, ${ }^{19}$ in which we only need to know the state of the system at each time step. We then try to modify it on-line, in order to take into account the modification of the external conditions.

In this paper, we propose to realize and compare two sets of adaptive closed-loop control experiments whose objective is to reduce the drag of an academic turbulent separated flow. We first describe the experimental setup, the measurement techniques, and the actuator that will be used in the control experiments. In Sec. III, we evaluate the effect of the actuator on the drag and try to analyze how it modifies the base flow. In Sec. IV, we propose a first step in closed-loop control using a proportional integral feedback law and demonstrate that this strategy allows our system to reach a predetermined set point in spite of external perturbations. In Sec. $\mathrm{V}$, we discuss the power balance of the system and use an extremum-seeking scheme ${ }^{20,21}$ in order to make our system

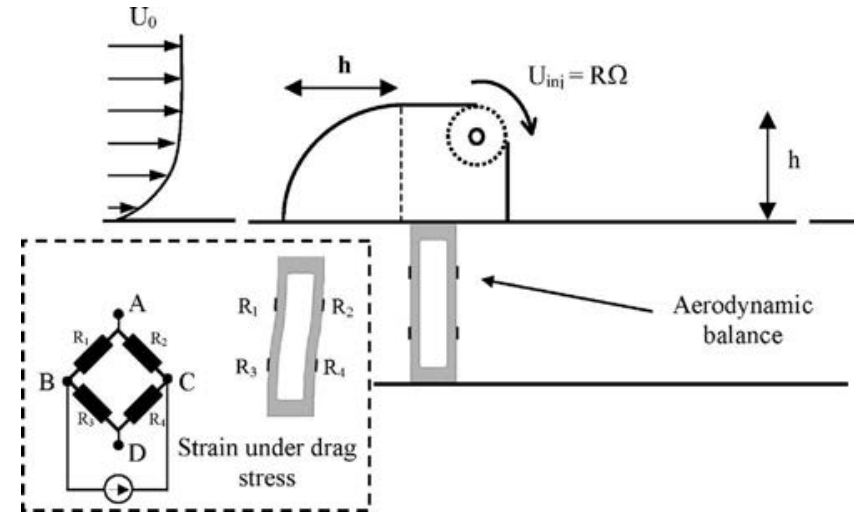

FIG. 1. Sketch of the experimental setup. A rotating cylinder located at the edge of the step modifies the characteristics of the flow separation. The system is placed on a plateau fixed to bimetallic brass strips on which four strain gauges are glued. The global strain is measured with a full Wheatstone bridge from Vishay (P 3100). The force (i.e., the drag) depends linearly on the strain. The full bridge configuration allows us to compensate temperature variations and to amplify the signal per a factor 2 compared to the half-bridge configuration. The output signal of the bridge is also amplified by a factor 10 giving a sensitivity of $0.0527 \mathrm{~N} / \mathrm{V}$, say $5.37 \mathrm{~g} / \mathrm{V}$.

able to optimize the global power autonomously and in real time, even with time-dependent flow conditions. Finally, we compare these two strategies and discuss our findings in Sec. VI.

\section{EXPERIMENTAL SETUP}

As we are interested in controlling separated flows, we choose to study the flow over a two-dimensional (2D) bluff body. The geometry is simple and consists in a quarter of a cylinder laying on the floor just in front of a square cylinder (Fig. 1). The height of the bluff body is $h=20 \mathrm{~mm}$ and its span length $100 \mathrm{~mm}$. We use a small open wind tunnel whose cross section is $100 \mathrm{~mm} \times 100 \mathrm{~mm}$. The upstream flow velocity $U_{0}$ ranges from 2 to $15 \mathrm{~m} \mathrm{~s}^{-1}$ so that the Reynolds number based on the bluff-body height $\operatorname{Re}=U_{0} h / \nu$ is between 3000 and 20000 .

This simple geometry creates a flow similar to the one behind a backward-facing step: the separation occurs at the edge and the flow reattaches further downstream. The separation surface is a region of strong unstable shears that are responsible for an important pressure drag.

We place a rotating cylinder at the edge of the backwardfacing step in order to modify the separation properties (Fig. 1). The present study only concerns positive rotation, i.e., when the cylinder forces the flow in the same direction as the main flow. The radius of the cylinder is $R=5 \mathrm{~mm}$ and the rotation rate $\Omega / 2 \pi$ can reach $250 \mathrm{~Hz}$ corresponding to a tangential velocity, or injection velocity, $U_{\text {inj }}=R \Omega$ up to $7.5 \mathrm{~m} \mathrm{~s}^{-1}$. To obtain significant drag reduction, the ratio $U_{\text {inj }} / U_{0}$ should be of the order 1 . The rotation of the cylinder is provided by a computer-driven dc motor, using LabView.

We evaluate the effect of the control on the drag using an aerodynamic balance composed of a plate mounted over a bimetallic brass strip (Fig. 1). Each strip is $0.3 \mathrm{~mm}$ thick and its behavior is similar to cantilever beams. The bluff body is maintained on the plate which is allowed to perform horizon- 


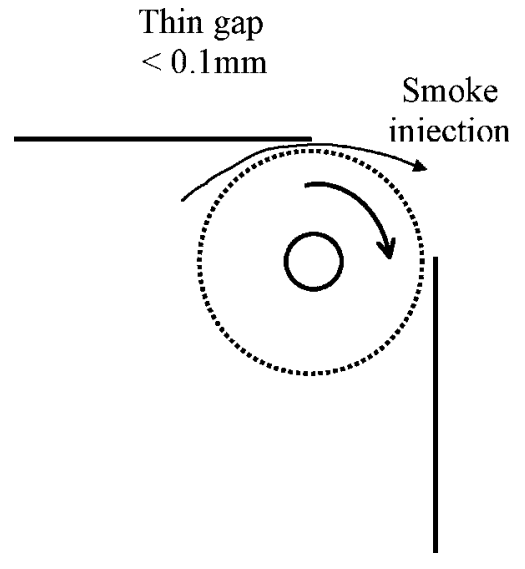

FIG. 2. Injection of cold smoke.

tal translations under a few tenths of a millimeter. When the flow is produced, the aerodynamic drag causes a translation of the plate, inducing a flexion of the sheets. Their deformation is measured by four strain gauges glued on the strip, providing a signal whose average value is proportional to the mean drag (see the inset in Fig. 1). The proper oscillation frequency of the brass structure is evaluated at $10.2 \mathrm{~Hz}$ (when loaded with the model) from analyzing its response to an impulse.

In order to understand the modification of the flow due to the control device, we perform laser-induced visualization in the symmetry plane of the bluff body. We inject cold smoke (a spray of $0.3 \mu \mathrm{m}$ di-ethyl-hexyl-sebacate droplets) into the bluff body itself (which is like an empty box) so that the smoke is naturally delivered into the flow through the thin gap (under $0.1 \mathrm{~mm}$ ) between the model and the cylinder (Fig. 2). This injection technique exhibits the recirculation zone very satisfactorily as displayed in Fig. 4(a). The images are captured with a CCD camera during a sufficiently long time (typically $1 / 30 \mathrm{~s}$, whereas typical shedding periods are about few milliseconds) so that we obtain a picture of the time-averaged flow.

We finally use hot-wire anemometry to obtain mean velocity profiles downstream from the step. Each acquisition lasts until statistical convergence of the mean quantities (typically a few tens of seconds).

\section{OPEN-LOOP CONTROL: INFLUENCE OF THE ACTUATOR ON THE BASE FLOW}

In this section, we study the effect of a uniform rotation of the cylinder on the drag and the mean flow structure and discuss the dependence of the results on two parameters, namely the mean flow velocity $U_{0}$ and the rotation rate $\Omega$.

\section{A. Drag reduction}

In Fig. 3(a), we show the evolution of the mean drag force $D$ as a function of the rotation rate of the cylinder $\Omega$ for three different free-stream velocities $U_{0}$. First we can notice that the rotation of the cylinder does not interact with the drag measurement. This is illustrated by the first curve (black squares), which corresponds to the case with no flow
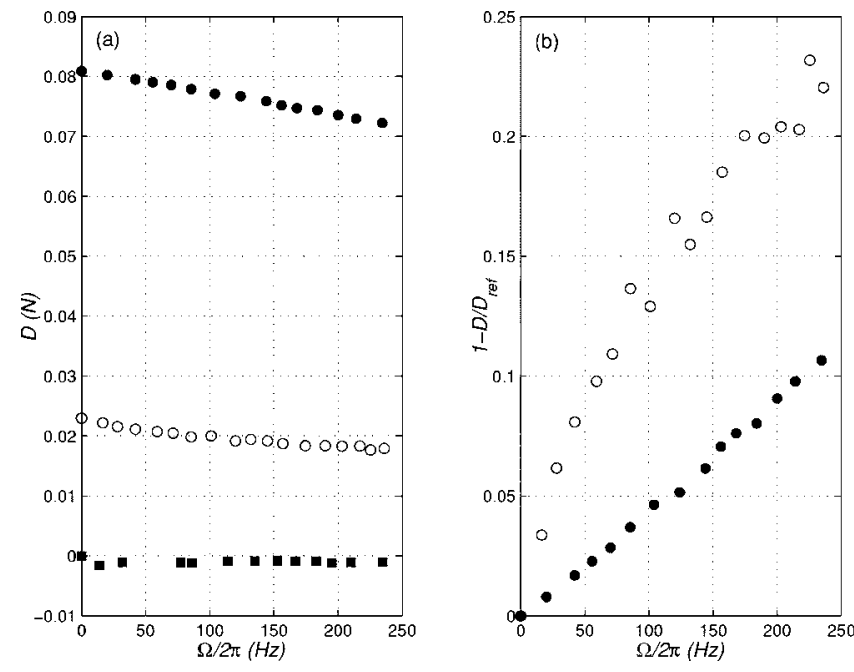

FIG. 3. Influence of $\Omega$ on the drag for various free-stream velocities $U_{0}$. (a) Drag measurements: black dots, $U_{0}=12 \mathrm{~m} \mathrm{~s}^{-1}$; white dots, $U_{0}=6 \mathrm{~m} \mathrm{~s}^{-1}$; black squares, no flow $\left(U_{0}=0 \mathrm{~m} \mathrm{~s}^{-1}\right)$. (b) Drag reduction rate: black dots, $U_{0}=12 \mathrm{~m} \mathrm{~s}^{-1}$; white dots, $U_{0}=6 \mathrm{~m} \mathrm{~s}^{-1}$.

$\left(U_{0}=0\right)$. We can see that the error is less than $10^{-3} \mathrm{~N}$, which is negligible when the free-stream velocity is high enough.

The other two curves show that for two different values of the free-stream velocity $\left(U_{0}=6\right.$ and $\left.12 \mathrm{~m} \mathrm{~s}^{-1}\right)$, the drag is a decreasing function of the rotation rate of the cylinder $\Omega$. The drag reduction rate, defined as $1-D / D_{\text {ref }}$ (with $D_{\text {ref }}$ the drag without control), is then an increasing function of $\Omega$ [Fig. 3(b)]. It reaches $23 \%$ and $11 \%$ for $U_{0}=6$ and $12 \mathrm{~m} \mathrm{~s}^{-1}$, respectively. One can notice that the relative drag gain is nearly proportional to the rotation rate when $U_{0}=12 \mathrm{~m} \mathrm{~s}^{-1}$ [Fig. 3(b)].

\section{B. Mean-flow modification}

Smoke visualizations of Fig. 4 are realized for $U_{0}=2 \mathrm{~m} \mathrm{~s}^{-1}$ and show clearly the difference between the natural separation [Fig. 4(a)] and the "controlled" separation [Fig. 4(b)]. The rotation of the cylinder clearly delays the

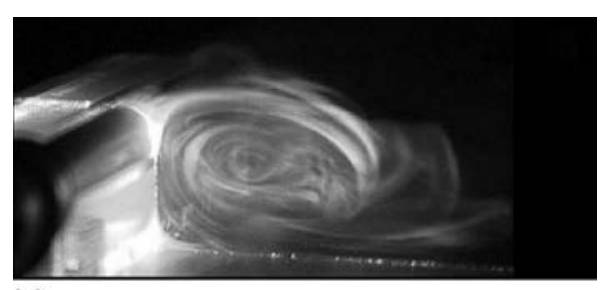

(a)

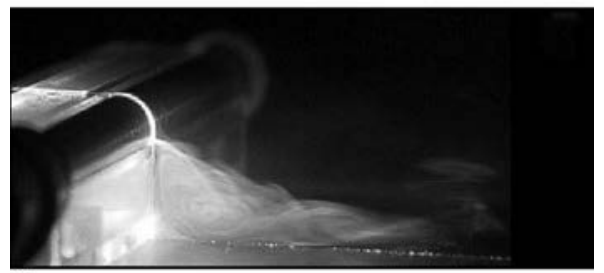

(b)

FIG. 4. Visualization of the separated flow downstream from the bluff body for $U_{0}=2 \mathrm{~m} \mathrm{~s}^{-1}$. (a) Without control; (b) with control $\left(U_{\mathrm{inj}} / U_{0}=2\right)$. 


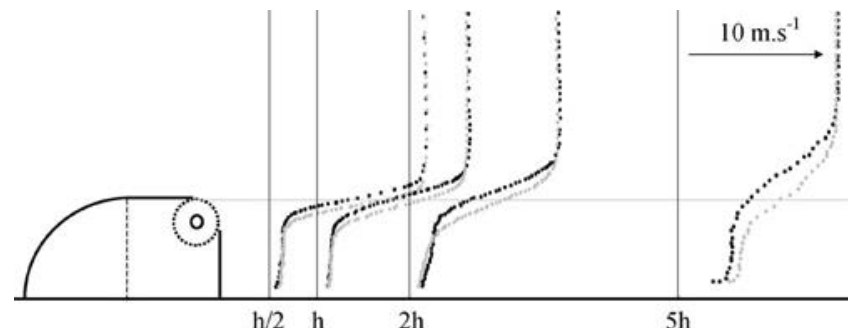

FIG. 5. Modulus of the mean velocity measured with hot wire anemometry for $U_{0}=12 \mathrm{~m} \mathrm{~s}^{-1}$. Black dots: without control. Gray dots: with control $\left(U_{\text {inj }} / U_{0}=0.5\right)$.

separation leading to a smaller recirculation bubble on the base of the bluff body and of course a smaller recirculation length.

In Fig. 5 we show the modification of the velocity profiles for higher free-stream velocity $\left(U_{0}=12 \mathrm{~m} \mathrm{~s}^{-1}\right)$, for which visualizations were not technically possible because of the turbulent diffusion of the smoke. The profiles exhibit the strong shear layer, which is clearly shifted downward when the cylinder is rotating (the black points, without control, are always above the gray points, with control). This is consistent with the fact that the separation point is also shifted downward. Hence, the results we obtain with hot wire anemometry at high velocities seem to confirm the observation we made with flow visualizations for lower velocities. As the ratio $U_{\mathrm{inj}} / U_{0}$ is much smaller for high free-stream velocities, the modification of the base flow is less spectacular than the one observed in the flow visualizations performed with $U_{0}=2 \mathrm{~m} \mathrm{~s}^{-1}$.

The drag reduction mechanism seems to be simple: the rotation of the cylinder moves the detachment point further downstream and provides a recovery of the pressure drop on the base of the bluff body, inducing a drag reduction. This is consistent with the experimental results of Munshi et al. ${ }^{4}$ showing the separation delay, pressure rise, and drag decrease obtained by momentum injection using sliding walls on the edges of rectangular prisms. As a matter of fact, the idea of using moving surfaces for boundary-layer separation control is not new: previous works ${ }^{4,13,22-24}$ are based on the hypothesis that a wall moving in the downstream direction causes a diminution of the relative motion between the wall and the free stream so that the boundary layer can undergo a more important adverse pressure gradient before breakdown. It is then an efficient tool for drag reduction or vortexinduced vibrations inhibition. ${ }^{4,13}$ Hence the originality of our study does not lie on the choice of this device, but on the fact that we directly evaluate its effect on the drag, and above all that we use it as a well-known actuator for closed-loop control experiments.

\section{PROPORTIONAL-INTEGRAL FEEDBACK CONTROL}

\section{A. Feedback scheme}

The objective of the closed-loop control is to make a dynamical system able to reach and keep a predetermined state in spite of external perturbations. One of the major

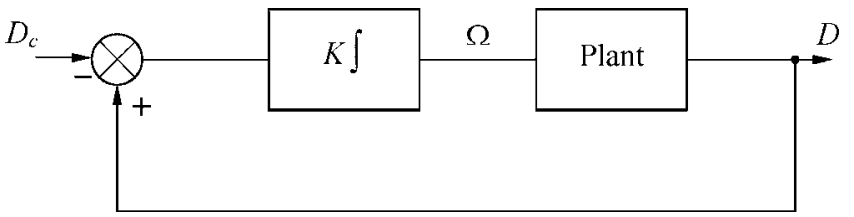

FIG. 6. Block-scheme of the closed-loop control. The drag measurement is used to give instruction to the motor.

difficulties is that measurements, information transfer, and activation of the actuator have to be simultaneously performed. In this section, we present a set of experiments that is a first step toward overcoming these difficulties.

We propose a closed-loop control allowing for the choice of a predetermined drag value for the system. Our experiment is characterized by a simple property of the drag evolution: it is a decreasing function of the cylinder rotation, $\frac{d D}{d \Omega}<0$.

We propose the following feedback law to control the rotation as a function of the drag measurement:

$$
\frac{d \Omega}{d t}=K\left[D(t)-D_{c}\right],
$$

where $D(t)$ is the measured drag, $D_{c}$ the chosen drag target value (i.e., a constant value the system must reach), and $K$ a positive gain adjusted by the experimentalist. As underlined by Eq. (2), the cylinder rotation is determined at each time step from the distance between the measured drag and its target value $D_{c}$,

$$
\Omega(t)=K \int\left[D(\tau)-D_{c}\right] d \tau .
$$

This feedback scheme is called proportional-integral because it consists in measuring the difference between the objective and the current state of the system, amplifying it before integrating it. The corresponding block-scheme is shown in Fig. 6.

It is possible to briefly justify the fact that such a closedloop system should converge writing $\frac{d D}{d t}=\frac{d D}{d \Omega} \frac{d \Omega}{d t}$. Since $D_{c}$ is constant in time, we can use the feedback law given by Eq. (1) to finally obtain

$$
\frac{d\left(D-D_{c}\right)}{d t}=K \frac{d D}{d \Omega}\left(D-D_{c}\right) \text {. }
$$

We then show that $\left(D-D_{c}\right)$ is governed by a first-order differential equation whose coefficient $K \frac{d D}{d \Omega}$ is negative, so that its solution should tend to 0 . Hence the measured $\operatorname{drag} D$ should reach its target value $D_{c}$.

\section{B. Experimental procedure}

In a typical experiment, we begin with choosing a target value $D_{c}$, which is the drag value the system must reach. In the definition of the loop [Eq. (1)], one can see that if the drag is higher than the target, the rotation speed will increase and then the drag will decrease; when the system reaches its objective, the acceleration of the motor vanishes and the system becomes stable. 


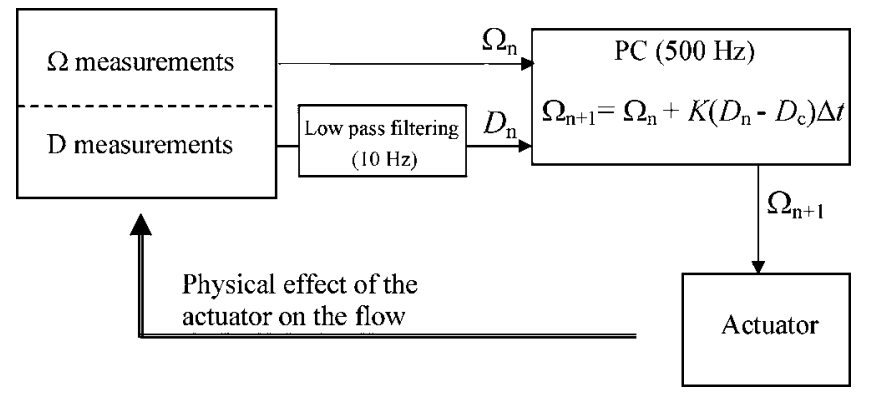

FIG. 7. Sketch of the experimental procedure used for the PID closed-loop control.

From a practical point of view, the control algorithm is insured by a PC under LabView software with a National Instrument board. This PC acquires both the drag and the rotation speed of the motor at the rate of $500 \mathrm{~Hz}$ in a single point acquisition mode. Each measured point corresponding to a time step $n, \Omega_{n}$, and $D_{n}$ are used for the numerical algorithm given by Eq. (4),

$$
\Omega_{n+1}=\Omega_{n}+K\left(D_{n}-D_{c}\right) \Delta t,
$$

where $\Delta t=t_{n+1}-t_{n}=2 \times 10^{-3} \mathrm{~s}$. The computed value of $\Omega_{n+1}$ is generated at one analog output channel of the board at the updated rate of $500 \mathrm{~Hz}$. This output is then used as the voltage consign for the dc motor. These steps are summarized in Fig. 7. Finally, we use a second PC only devoted to the acquisition of both signals, $\Omega(t)$ and $D(t)$ in a buffer acquisition mode at a sample frequency of $1 \mathrm{kHz}$.

\section{Results and discussion}

The data discussed in this section are obtained by starting the recording without control, before starting the control a few seconds later. Without loss of generality, we only consider the case $U_{0}=12 \mathrm{~m} \mathrm{~s}^{-1}$ and report results for various values of the gain $K$. We show in Figs. 8-10 time series of drag normalized by its reference value $D / D_{\text {ref }}$, and rotation fluctuations.
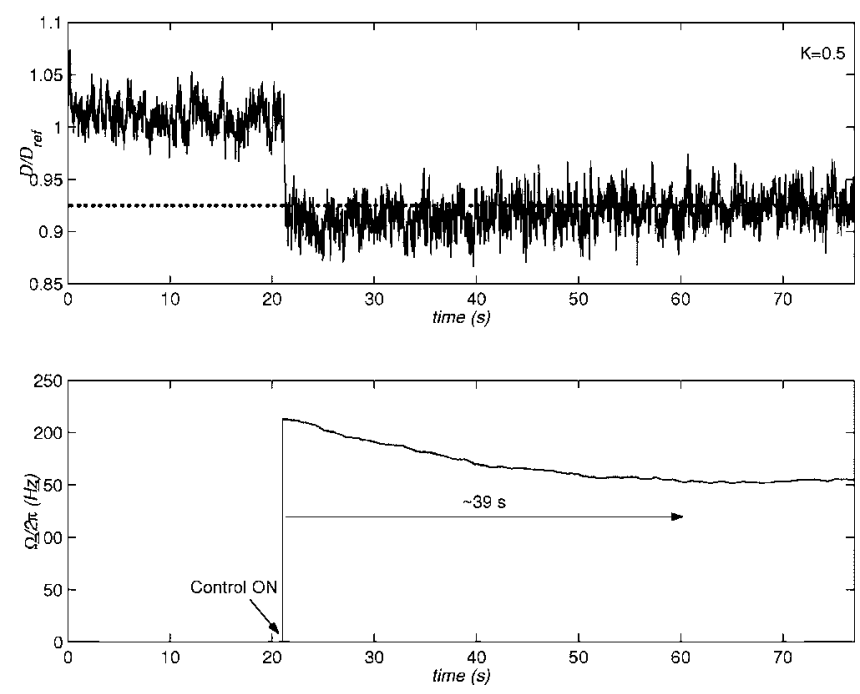

FIG. 8. Time series for $K=0.5$ (arbitrary units). Upper figure: $D / D_{\text {ref }}$, full line; $D_{c}$, dashed line. Lower figure: rotation frequency $\Omega$.
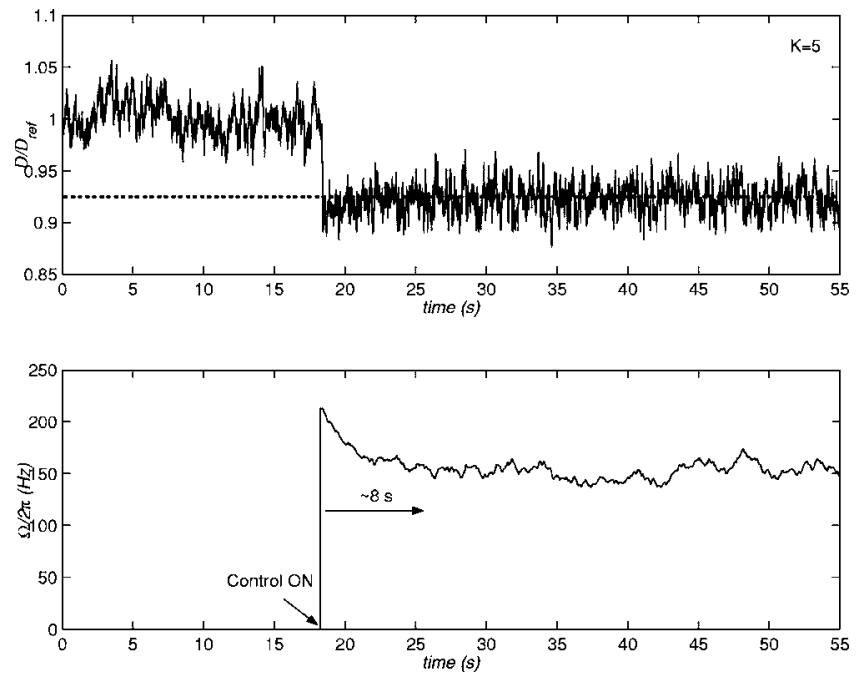

FIG. 9. Time series for $K=5$ (arbitrary units). Upper figure: $D / D_{\text {ref }}$, full line; $D_{c}$, dashed line. Lower figure: rotation frequency $\Omega$.

At the beginning of each time series, there is no rotation and $D$ is normally equal to its reference value $D_{\text {ref. }}$ As soon as the closed-loop control is released, the system becomes autonomous and we observe that $\Omega$ increases sharply and goes above the objective $D_{c}$ before decreasing regularly, reaching the objective and stabilizing around it. In all cases, the rotation speed converges to $150 \mathrm{~Hz}$, which corresponds to the rotation we should impose in the open-loop case to reach a 7\% drag reduction (Fig. 3). The stabilization time is clearly dependent on the gain parameter $K$. By comparing the first two experiments, we can notice that as $K$ increases from 0.5 to 5 , the system becomes stable more quickly (stabilization time decreases from 39 to $8 \mathrm{~s}$ ). This observation is consistent with the previous discussion concerning the system convergence, as we can see in Eq. (3) that the higher $K$ is, the faster is the convergence. We also have to notice that
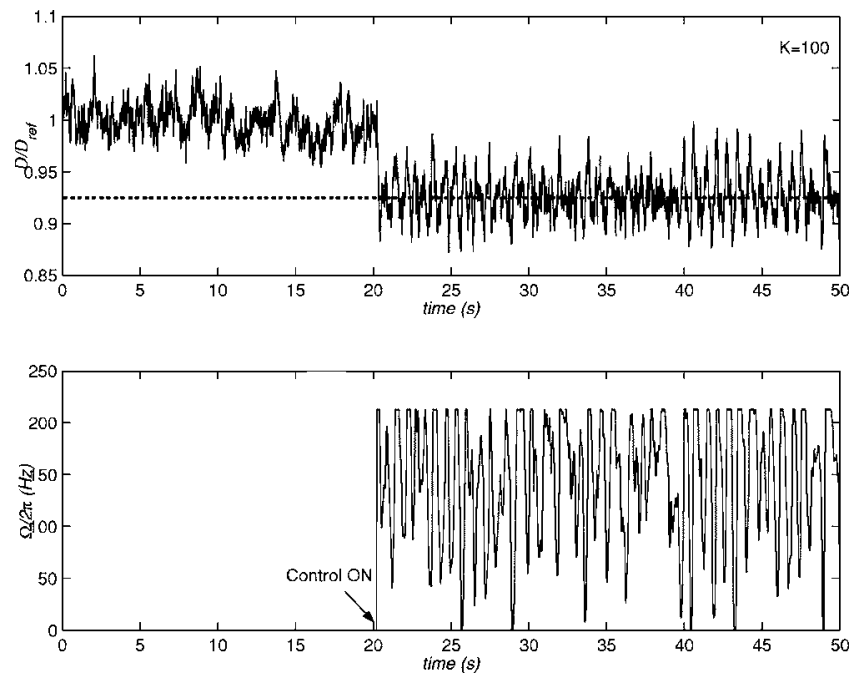

FIG. 10. Time series for $K=100$ (arbitrary units). Upper figure: $D / D_{\text {ref }}$, full line; $D_{c}$, dashed line. Lower figure: rotation frequency $\Omega$. 
for higher $K$ values (Fig. 10) the system still reaches its objective but experiences very strong rotation fluctuations, due to a lack of stability.

This set of experiments shows that it is possible to create an experimental system able to reach autonomously a predefined stable state. It is also interesting to notice that it is in good agreement with the numerical experiment of Patnaik and $\mathrm{Wei}^{13}$ studying the control of a square cylinder wake by momentum injection at the edges (in a laminar case). Their closed-loop scheme is similar to the proportional-integral algorithm and is based on the measurements of the velocity fluctuations in the whole domain, which is an integral quantity, as the drag force. In Ref. 13, the behavior of the rotation and that of the state of the system are similar to the behavior we report in this article, which underlines the encouraging results we obtained in the case of turbulent flows.

Nevertheless, one of the main limitations of the method is that by construction, the objective is imposed by the experimentalist and must be chosen a priori. It is then important to search for new algorithms, more complex and more robust, allowing the system to identify and reach its final state without any previous instructions. This is the purpose of the following section.

\section{REAL-TIME MINIMIZATION OF THE GLOBAL POWER OF THE SYSTEM}

\section{A. Power balance}

By definition, active control requires an external energy input to activate the control device. It is then essential for the control to be useful that the actuation does not use more energy than the system gains thanks to the control: the power balance (difference between gain and loss of energy) must be positive. In our case, we want the highest drag reduction to reduce the dissipation through aerodynamic power $\mathcal{P}_{a}=D U_{0}$. To reach this goal, we have to use electric power $\mathcal{P}_{e}$ for the rotation of the actuator. The aerodynamic power $\mathcal{P}_{a}$ depends on both the rotation speed $\Omega$ and the free-stream velocity $U_{0}$ (see Fig. 3), while the electric power $\mathcal{P}_{e}$ is found to only depend on $\Omega$ (aerodynamic friction being negligible before solid friction). The measured electric power $\mathcal{P}_{e}=V I$, where $V$ and $I$ are, respectively, the voltage and the current applied to the dc motor, can be fitted by a quadratic law, $\mathcal{P}_{e}=\alpha \Omega^{2}$ (Fig. 11). In the following, the electric power is always estimated from the angular velocity of the dc motor using this fitted law. We can then define the power balance function $J\left(\Omega, U_{0}\right)$ as the sum of electric and aerodynamic power,

$$
J\left(\Omega, U_{0}\right)=\mathcal{P}_{a}\left(\Omega, U_{0}\right)+\mathcal{P}_{e}(\Omega)=D\left(\Omega, U_{0}\right) U_{0}+\alpha \Omega^{2} .
$$

From the experimental point of view, the instantaneous $J$ function as defined in (5) is computed on a PC using a single-point acquisition mode of the rotation frequency $\Omega$, the free-stream velocity $U_{0}$, and the drag measurements $D$ with a $200 \mathrm{~Hz}$ update rate.

As the aerodynamic power is a decreasing function of the rotation frequency $\Omega$ (Fig. 3) and the electric power an increasing function of $\Omega$ (Fig. 11), the $J(\Omega)$ function must have a minimum for all free-stream velocities. We measured

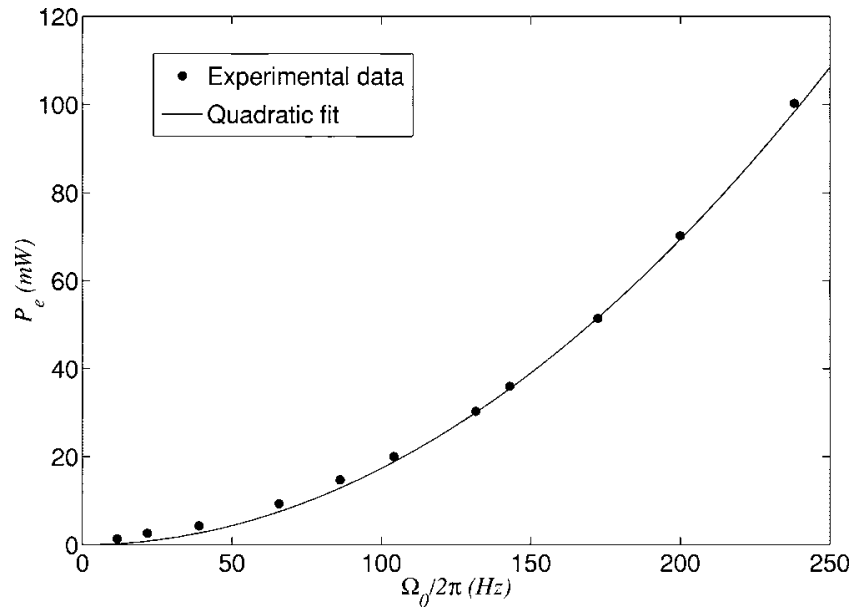

FIG. 11. Electric power used by the motor as a function of the rotation frequency: measurements (black points) and quadratic fit (continuous line) $\mathcal{P}_{e}=\alpha \Omega^{2}$, where $\alpha=4.44 \times 10^{-8} \mathrm{~W} /\left(\operatorname{rad~s}^{-1}\right)^{2}$.

the averaged value of the $J$ function as a function of $\Omega$ for a given free-stream velocity $U_{0}=12 \mathrm{~m} / \mathrm{s}$. We obtain the curve shown in Fig. 12, which clearly has a minimum, even if poorly defined, between 100 and $150 \mathrm{~Hz}$. For this magnitude of rotational frequencies, the drag reduction is about $5 \%$, as can be seen in Fig. 3(b). The objective of the closedloop experiment is then for the system to reach autonomously this minimum. We should then be able to use an "extremum-seeking control" strategy to help the system to find its minimum.

\section{B. Extremum-seeking control scheme and experimental procedure}

In the previous section, we defined our new objective: to minimize the total power dissipated by the system for any free-stream velocity $U_{0}$. The control parameter $\Omega$ must be

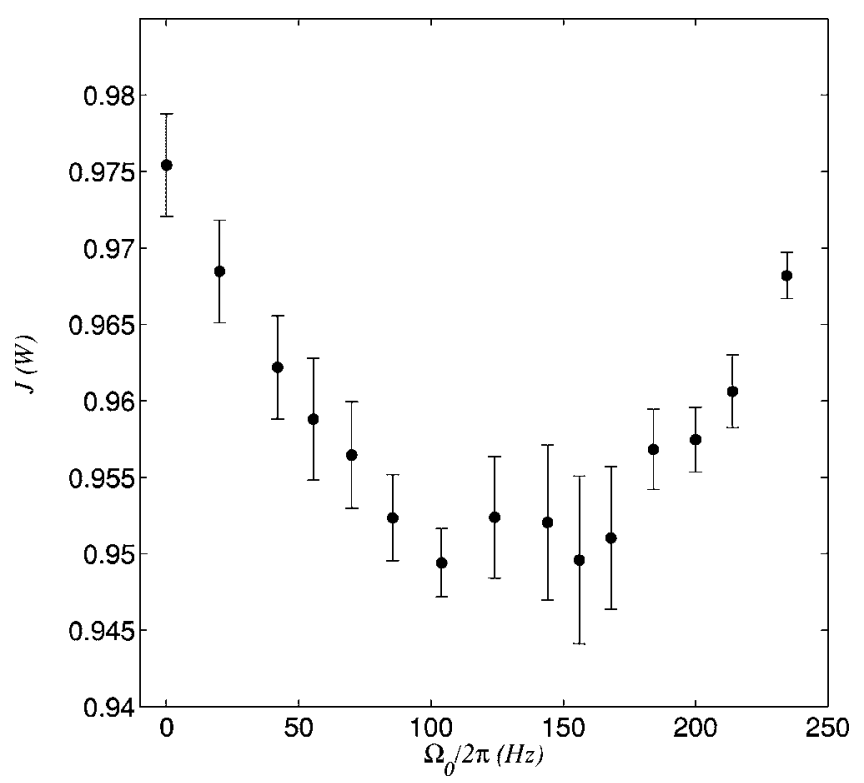

FIG. 12. Evolution of $J$ as a function of the rotation speed $\Omega$ for $U_{0}=12 \mathrm{~m} / \mathrm{s}$. 
adjusted by the system to reach the state of minimum power injected corresponding to the minimum of the $J$ function. In this respect, we choose to use an "extremum-seeking control" strategy that is efficient in the case in which a nonlinear plant has an extremum. It consists in using a real-time gradient optimization method with the following feedback law:

$$
\frac{d \Omega}{d t}=-K \frac{\partial J}{\partial \Omega},
$$

where $K$ is a positive gain that is chosen by the experimentalist.

In the case of turbulent flows, the fluctuations of physical quantities are large with a broad spectrum. This situation makes the evaluation of the gradient by classic finite difference nearly impossible. Krstić and Wang ${ }^{21}$ have recently revisited the sinusoidal perturbations method, which allows us to deal with largely fluctuating signals. It consists in modulating the input of our system in the following way:

$$
\Omega(t)=\Omega_{0}(t)+a \cos \left(2 \pi f_{m} t\right) .
$$

From now on, $\Omega$ is no longer constant, but is modulated around $\Omega_{0}(t)$. The modulation amplitude $a$ and frequency $f_{m}$ are chosen by the experimentalist and are constant during the experiments. The constant $a$ has to be sufficiently large and $f_{m}$ lower than the cutoff frequency of the balance to detect the effect of the modulation on the drag measurements. We then took $a / 2 \pi=40 \mathrm{~Hz}$ and $f_{m}=1 \mathrm{~Hz}$. The objective is that $\Omega_{0}(t)$, which is a slowly variable function of time, reaches the value corresponding to the minimum of the $J$ function, regardless of the free-stream velocity $U_{0}$. We will speak about modulated open loop when the rotation is modulated and $\Omega_{0}$ constant in time.

To estimate the gradient of $\frac{\partial J}{\partial \Omega}\left(\Omega_{0}\right)$, a second PC acquires $J(t)$ (from the first PC) and $\Omega(t)$ in a single-point acquisition mode at a sampling rate of $50 \mathrm{~Hz}$. This PC performs fast Fourier transform of both quantities over a sliding window of 4096 points corresponding to a $81.92 \mathrm{~s}$ time window. A temporal window of $J$ and its amplitude spectrum is displayed in Fig. 13 during modulated open loop. The spectrum of $J$ is dominated by the drag measurements. The narrow peak at $10 \mathrm{~Hz}$, corresponding to the lowest-frequency mode of the balance, is followed by a wide peak. We do not have any clear idea about the origin of this wide peak that could correspond other modes of the balance. However, the presence of these peaks is not a limitation since we are interested in the measurements at lower frequencies that are the mean and the amplitude at the modulation frequency. The modulation peak at $f_{m}=1 \mathrm{~Hz}$ is clearly distinguishable (modulated peak in Fig. 13). Both the amplitude $A_{J}\left(f_{m}\right)$ and the phase $\phi_{J}\left(f_{m}\right)$ of the mode $f_{m}$ are then extracted from the spectrum of the sliding windows. The values are updated at a rate of $50 \mathrm{~Hz}$. Simultaneously, the same treatment is performed on $\Omega(t)$ in order to extract its phase $\phi_{\Omega}\left(f_{m}\right)$ at the modulation frequency. The relationship between these measured quantities and the gradient is the following. The development of the $J$ function around the actual value $\Omega_{0}$ reads
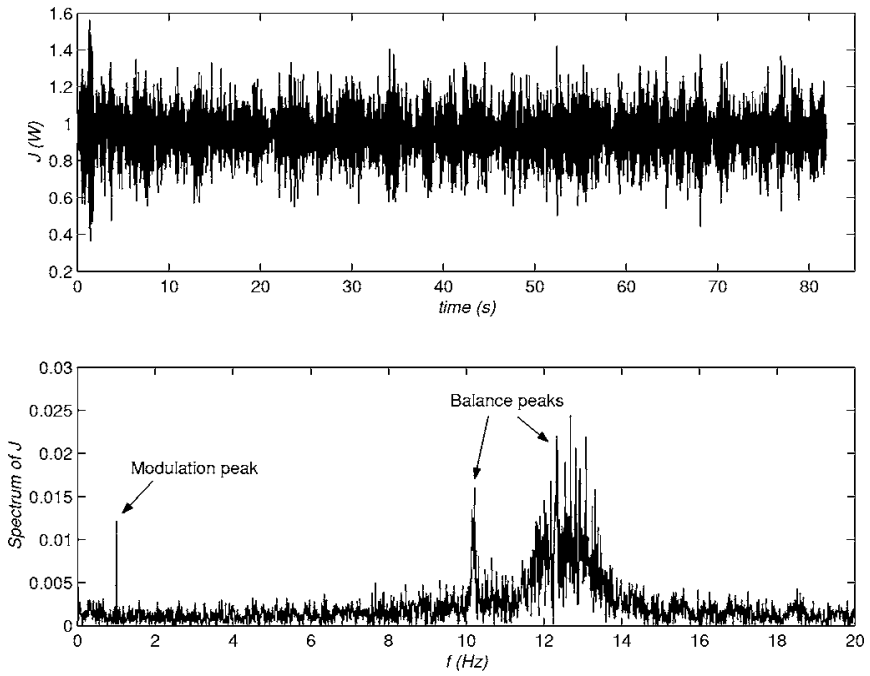

FIG. 13. Upper figure: typical time series of the $J$ function. Lower figure: $J$ spectrum of the time series computed on a 4096-point sliding window. The modulation peak corresponding to the modulation frequency $f_{m}=1 \mathrm{~Hz}$ is clearly identified in the amplitude spectrum of $J$.

$$
J(\Omega)=J\left(\Omega_{0}\right)+\left(\Omega-\Omega_{0}\right) \frac{\partial J}{\partial \Omega}\left(\Omega_{0}\right) .
$$

Since $\Omega$ is modulated as in Eq. (7), the response of the $J$ function to the modulation is for $a$ small enough,

$$
\begin{aligned}
J\left[\Omega_{0}+a \cos \left(2 \pi f_{m} t\right)\right]= & J\left(\Omega_{0}\right)+a \cos \left(2 \pi f_{m} t+\varphi_{\pi}\right) \\
& \times\left\|\frac{\partial J}{\partial \Omega}\left(\Omega_{0}\right)\right\|,
\end{aligned}
$$

with $\varphi_{\pi}=0$ or $\pi$. In this case $J$ is also a harmonic function of time whose amplitude of the mode $f_{m}$ is proportional to $\left\|\frac{\partial J}{\partial \Omega}\left(\Omega_{0}\right)\right\|$. Also, the phase shift $\varphi_{\pi}=\phi_{\Omega}\left(f_{m}\right)-\phi_{J}\left(f_{m}\right)$, being the phase difference between both modes of $\Omega$ and $J$ at the frequency $f_{m}$, gives the sign of the gradient, and we have

$$
\frac{\partial J}{\partial \Omega} \approx \frac{A_{J}\left(f_{m}\right)}{a} \operatorname{sgn}\left\{\cos \left[\phi_{\Omega}\left(f_{m}\right)-\phi_{J}\left(f_{m}\right)\right]\right\} .
$$

Finally the second PC gives 50 orders per second to the motor controlling the rotation of the cylinder according to the following relation:

$$
\begin{aligned}
& \Omega_{n+1}=\Omega_{0, n+1}+a \cos \left[2 \pi f_{m}(n+1) \Delta t\right], \\
& \Omega_{0, n+1}=\Omega_{0, n}-K \frac{A_{J}\left(f_{m}\right)}{a} \operatorname{sgn}\left\{\cos \left[\phi_{\Omega}\left(f_{m}\right)-\phi_{J}\left(f_{m}\right)\right]\right\} \Delta t,
\end{aligned}
$$

where $\Delta t=t_{n+1}-t_{n}=2 \times 10^{-2} \mathrm{~s}$. More details about this technique are given in Refs. 20 and 21. The different steps of this closed-loop control algorithm are summarized for its principle in the block diagram in Fig. 14 and for its experimental procedure in Fig. 15.

In order to characterize the robustness of the gradient estimator, we recorded $J$ and $\operatorname{sgn}(\cos \varphi) A_{J}\left(f_{m}\right)$ during $100 \mathrm{~s}$ in an open-loop control experiment (see Fig. 13). We repeated this recording for different rotation frequencies $\Omega_{0}$ of the actuator. We display the average of $J$ together with 


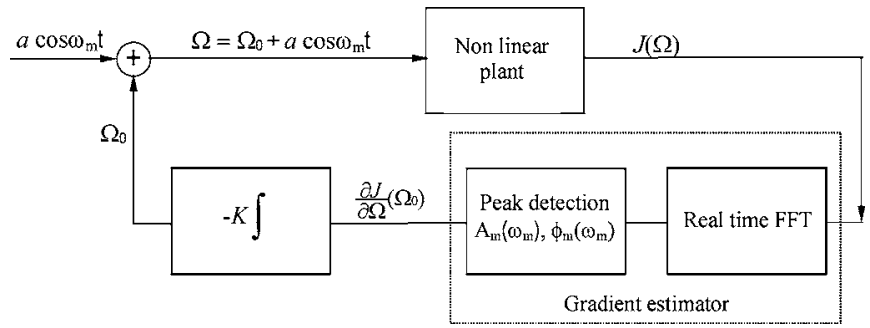

FIG. 14. Sketch of the feedback closed-loop control using the sinusoidal perturbations method.

$\operatorname{sgn}(\cos \varphi) A_{J}\left(f_{m}\right)$ in Fig. 16. The measurements are accompanied with error bars that correspond to their root-mean square values. We can check that the algorithm is able to detect the minimum of $J$. However, the gradient magnitude is not well estimated around this minimum where it should go to 0 .

\section{Results and discussion}

In a first experiment, we use the experimental procedure described in Fig. 15 and the relation (12), with $U_{0}=12 \mathrm{~m} / \mathrm{s}$ (Fig. 17). At the beginning of the experiment, there is no forcing $(\Omega=0)$ and $J=J_{\text {ref }} \simeq 0.98 \mathrm{~W}$. After some times, we apply the modulated open-loop control with $\Omega_{0}=45 \mathrm{~Hz}$ and observe the decreasing (in less than $80 \mathrm{~s}$ ) of the $J$ function toward a value close to $0.96 \mathrm{~W}$, which corresponds to the mean value we measured in open-loop control for $\Omega_{0}=45 \mathrm{~Hz}$ (Fig. 12). Then we trigger the closed-loop control and observe a new decrease of $J$, which finally begins to oscillate around a mean value close to $0.95 \mathrm{~W}$. During this last step, the system is completely autonomous and no longer needs any external intervention. The mean value of the rotation $\Omega_{0}$ increases as soon as the loop is closed and oscillates around $100 \mathrm{~Hz}$. One can underline that the mean value $J$ corresponding to $\Omega_{0}=100 \mathrm{~Hz}$ in the open-loop experiment is about $0.95 \mathrm{~W}$, corresponding to the second plateau of the $J$ function (Fig. 12). The large fluctuations of $\Omega_{0}$ can be explained by the fact that the minimum of the $J$ function is not well defined.

We now perform the same experiment with a slight difference: after some times, we modify the free-stream veloc-

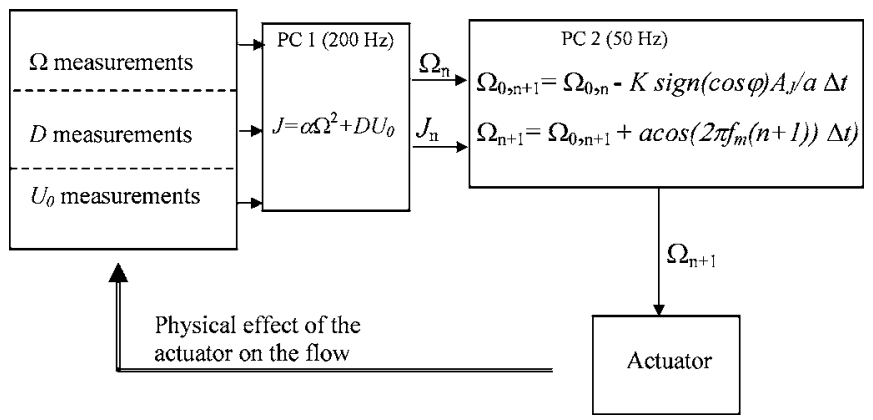

FIG. 15. Description of the experimental procedure used for closed-loop control using the sinusoidal perturbations method.

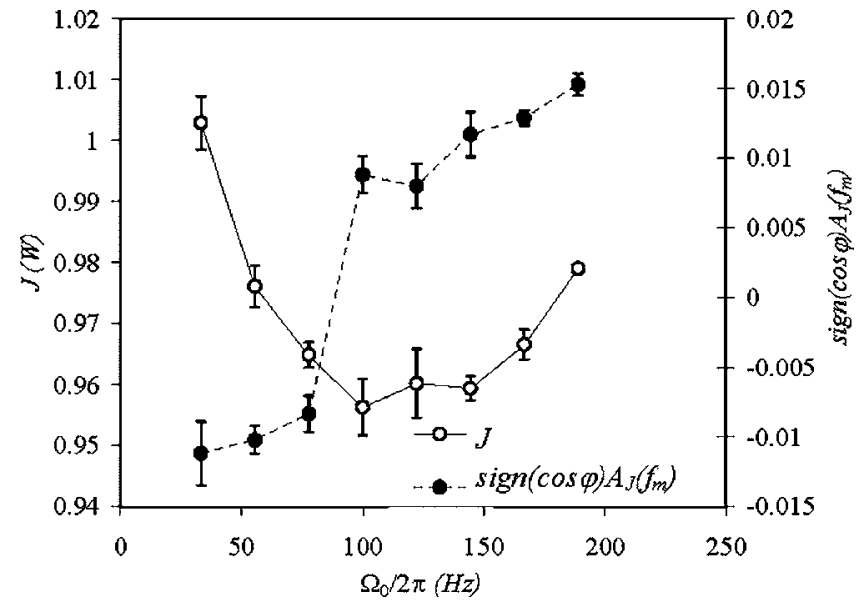

FIG. 16. Open-loop control results about estimation of the gradient of $J$ versus $J$ at the mean rotation frequency $\Omega_{0}$ of the actuator.

ity to check if the system is able to fit to these changing flow conditions and can identify and reach its new optimal state without any external intervention.

The experiment begins with no forcing (Fig. 18) leading to $J=J_{\text {ref }} \simeq 0.98 \mathrm{~W}$. After a short time of modulated openloop control (with $\Omega_{0}=45 \mathrm{~Hz}$ ), we start the extremumseeking control loop. Just like in the previous experiment, $J$ reaches the value $0.95 \mathrm{~W}$ while $\Omega_{0}$ increases before fluctuating around the mean value $\Omega_{0}=100 \mathrm{~Hz}$. After a $1600 \mathrm{~s}$ recording, the free-stream velocity is lowered from $U_{0}=12 \mathrm{~m} / \mathrm{s}$ to $U_{0}=10 \mathrm{~m} / \mathrm{s}$ (Fig. 18) so that the drag decreases sharply and, consequently, $J=D U_{0}+\mathcal{P}_{e}$ strongly decreases. The loop is still closed and the system reaches autonomously a new mean rotation speed $\Omega_{0}=70 \mathrm{~Hz}$ around which it fluctuates. The value of the $J$ plateau is about $0.50 \mathrm{~W}$. Finally $\Omega$ is set to 0 to turn the forcing off. $J$ increases and reaches again its reference value about $0.51 \mathrm{~W}$ for $U_{0}=10 \mathrm{~m} / \mathrm{s}$. The corresponding value of the $J$ plateau is about $0.51 \mathrm{~W}$. This new plateau is higher than the value
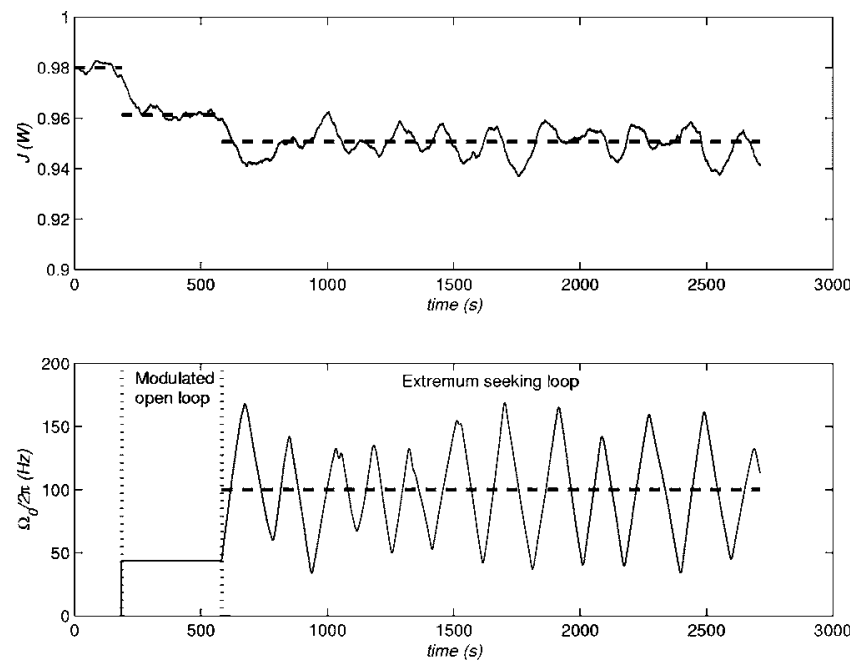

FIG. 17. Open-loop and closed-loop control experiment for $U_{0}=12 \mathrm{~m} / \mathrm{s}$. Upper figure: $J$, fine continuous line; plateau of $J$, dashed line. Lower figure: rotation $\Omega_{0}$, continuous line; plateau of $\Omega_{0}$, dashed line. 

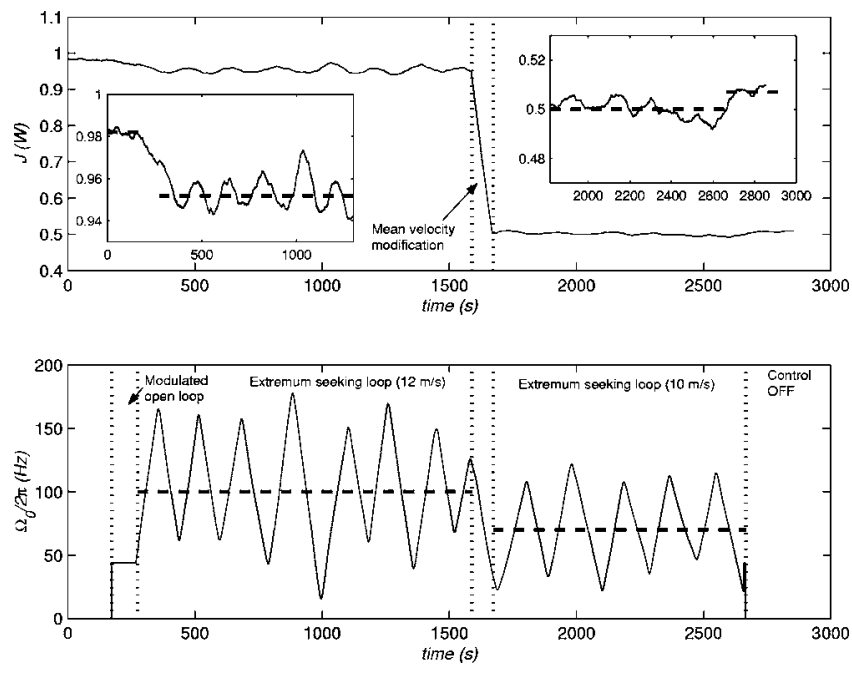

FIG. 18. Open-loop and closed-loop control experiment with a change of the free-stream velocity during the experiment (from $U_{0}=12 \mathrm{~m} / \mathrm{s}$ to $U_{0}=10 \mathrm{~m} / \mathrm{s}$ ). Upper figure: $J$, continuous line; $J$ plateau, dashed line (the two windows in the main window are an enlargement of the beginning and the end of the signal). Lower figure: rotation $\Omega_{0}$, continuous line; $\Omega_{0}$ plateau, dashed line.

reached by the system in closed loop, which is the evidence of the efficiency of the loop, even if we cannot assert that it is exactly the optimal state.

The experimental results reported in this section show that the extremum-seeking closed-loop control strategy based on the sinusoidal perturbations method is efficient, using our experimental procedure. In both experiments it makes the system able to evaluate its global power budget in real time (through the measurements of the $J$ function) and then to identify and reach its optimal set point, which corresponds to the maximum power saving, without any external intervention. Finally, it appears that this adaptive control method allows the system to fit to variable flow conditions, such as the mean-flow velocity.

\section{CONCLUDING REMARKS}

In this article, we report experimental results concerning open- and closed-loop control of a bi-dimensional turbulent separation, in order to reduce the drag of a bluff body. We first demonstrate the efficiency of moving walls to delay separation and then reduce the drag. The effect of a rotating cylinder placed on the edge of the bluff body is larger as the rotation is increased, which allows remarkable actuation for closed-loop experiments.

Next we try a proportional-integral algorithm to impose a predefined state on the system. In this case, the experimentalist chooses a target value the system should reach and the drag measurement is used to build the instructions given to the motor. Using our experimental procedure, the system is actually able to reach its objective.

Then we propose an energy condition so that the system will search for the optimal state corresponding to the compromise between the maximum drag reduction and the minimum electric power consumption. In this second set of experiments, we propose a real-time gradient method that can be applied thanks to a sinusoidal perturbations method. It consists in modulating the input of the system before performing a spectral analysis of the output in order to evaluate the gradient of the function we want to optimize. Using the estimated gradient, we modify the input in real time so that the system reaches and oscillates around its optimal state. We demonstrate the efficiency of the algorithm by performing an experiment with time-dependent external conditions: the free-stream velocity is modified and the system reaches autonomously its new optimal state.

Both of the adaptive methods that are detailed in this study seem to be efficient since they make the system able to react in real time. As the proportional-integral algorithm requires little data processing, it is a rather fast method. But its main limitation lies in the fact that the set point the system reaches has to be defined a priori. On the contrary, the extremum-seeking scheme allows the system to identify its objective by its own in the general case in which a nonlinear plant presents an optimum. Since in this case the data processing is rather important (in particular because of the spectral analysis), this closed loop is much slower than the first one, but appears to be efficient.

From an academic point of view, we emphasize the fact that not many experiments in fluid mechanics attest to the real efficiency of active control in globally reducing the energy consumption of a system. In this sense, our experiment is a very convincing illustration.

As a result, we believe that both of these methods are interesting for other applications and we demonstrate that they overcome the main difficulties associated with the closed-loop control of turbulent flows. Hence we are confident that such adaptive schemes could be a powerful tool for some industrial applications. In particular, Beaudoin ${ }^{25}$ has recently used the same extremum-seeking control experimental procedure to reduce the drag of a simplified vehicle (with a totally different type of actuator) with success.

\section{ACKNOWLEDGMENTS}

We would like to thank P. Rouchon and B. Protas for fruitful discussions. We also acknowledge the useful help of D. Pradal and D. Vallet in preparing the model for the experiments.

${ }^{1}$ T. R. Bewley, "Flow control: New challenges for a new Renaissance," Prog. Aerosp. Sci. 37, 21 (2001).

${ }^{2}$ M. Gad-El-Hak, "Flow control," Appl. Mech. Rev. 42, 261 (1989).

${ }^{3}$ M. Gad-El-Hak and D. M. Bushnell, "Separation control: Review," J. Fluids Eng. 113, 5 (1991).

${ }^{4}$ S. R. Munshi, V. J. Modi, and T. Yokomizo, "Aerodynamics and dynamics of rectangular prisms with momentum injection," J. Fluids Struct. 11, 873 (1997).

${ }^{5}$ B. Protas and J. E. Wesfreid, "Drag force in the open-loop control of the cylinder wake in the laminar regime," Phys. Fluids 14, 810 (2002).

${ }^{6}$ D. Greenblatt and I. J. Wygnanski, "The control of flow separation by periodic excitation," Prog. Aerosp. Sci. 36, 487 (2000).

${ }^{7}$ T. R. Bewley, P. Moin, and R. Temam, "DNS-based predictive control of turbulence: An optimal benchmark for feedback algorithms," J. Fluid Mech. 447, 179 (2001). 
${ }^{8}$ H. Choi, P. Moin, and J. Kim, "Active turbulence control for drag reduction on wall-bounded flows," J. Fluid Mech. 262, 75 (1994).

${ }^{9}$ N. Fujisawa, Y. Kawaji, and K. Ikemoto, "Feedback control of vortex shedding from a circular cylinder by rotational oscillations," J. Fluids Struct. 15, 23 (2001).

${ }^{10}$ N. Fujisawa and T. Nakabayashi, "Neural network control of vortex shedding from a circular cylinder using rotational feedback oscillations," J. Fluids Struct. 16, 113 (2002).

${ }^{11}$ M. Garwon, L. H. Darmadi, F. Urzynicok, G. Bärwolff, and R. King, "Adaptive control of separated flows," in Proceedings of the 2003 European Control Conference, Cambridge, UK (2003).

${ }^{12}$ J. Kim, "Control of turbulent boundary layers," Phys. Fluids 15, 1093 (2003).

${ }^{13}$ B. S. V. Patnaik and G. W. Wei, "Controlling wake turbulence," Phys. Rev. Lett. 88, 054502 (2002).

${ }^{14}$ B. Protas and A. Styczek, "Optimal rotary control of the cylinder wake in the laminar regime," Phys. Fluids 14, 2073 (2002).

${ }^{15}$ M. M. Zhang, L. Cheng, and Y. Zhou, "Closed-loop-controlled vortex shedding and vibration of a flexibly supported square cylinder under different schemes," Phys. Fluids 16, 1439 (2004).

${ }^{16}$ Y. Wang, G. Haller, A. Banaszuk, and G. Tadmor, "Closed-loop Lagrang- ian separation control in a bluff body shear flow model," Phys. Fluids 15, 2251 (2003).

${ }^{17}$ C. M. Ho and Y. C. Tai, "Review: MEMS and its applications for flow control," J. Fluids Eng. 118, 437 (1996).

${ }^{18}$ C. M. Ho and Y. C. Tai, "Micro-electro-mechanical-systems (MEMS) and fluid flows," Annu. Rev. Fluid Mech. 30, 579 (1998).

${ }^{19}$ M. Krstić, I. Kanellakopoulos, and P. V. Kokotovic, Nonlinear and Adaptive Control Design (Wiley, New York, 1995).

${ }^{20}$ J. F. Beaudoin, O. Cadot, J. L. Aider, and J. E. Wesfreid, "Bluff-body drag reduction by extremum seeking control," J. Fluids Struct. (in press).

${ }^{21}$ M. Krstić and H. H. Wang, "Stability of extremum seeking feedback for general nonlinear dynamic systems," Automatica 36, 595 (2000).

${ }^{22}$ A. Alvarez-Calderon, "Rotating cylinder flaps of V/STOL aircraft," Aircr. Eng. 36, 304 (1964).

${ }^{23}$ B. Choi and H. Choi, "Drag reduction with a sliding wall in flow over a circular cylinder," AIAA J. 38, 715 (1999).

${ }^{24}$ V. J. Modi, F. Mokhtarian, M. S. U. K. Fernando, and T. Yokomizo, "Moving surface boundary-layer control as applied to two-dimensional airfoils," J. Aircr. 28, 104 (1991).

${ }^{25}$ J. F. Beaudoin, "Contrôle actif d'écoulement en aérodynamique automobile," Ph.D. thesis, Ecole des Mines de Paris (2004). 